\title{
APPLICATION OF PATH PLANNING FOR INTELLIGENT MECHANICAL ROBOT UNDER EMBEDDED REAL-TIME SYSTEM
}

\author{
Yunfei Wang ${ }^{1}$, Mingliang Liang ${ }^{1}$ \\ ${ }^{1}$ Zhengzhou Railway Vocational\&Technical College, Zhengzhou, 451460, He'nan, China. \\ Email:wangyunfeiwfyls@126.com
}

\begin{abstract}
Objective: The paper is to study the path planning of the intelligent mechanical robot under the embedded real-time system, to provide theoretical support for the path planning problems of mobile robots. Method: In this paper, the embedded system is applied to the path planning of the mobile robot, and it is used as the processor of the mobile robot. The path planning of the mobile robot and algorithms to avoid obstacles are also studied. Also, this paper proposes an improved $\mathrm{A}^{*}$ algorithm. It will build a specific environment grid based on the existing environment materials and use the improved $A^{*}$ algorithm to analyze each node in detail to explore the most suitable and fastest path. Results: The total turning angle of the mobile robot using the primary $\mathrm{A}^{*}$ algorithm is $180^{\circ}$ and the path length is $10.07 \mathrm{~m}$. The total turning angle of the mobile robot using the secondary $\mathrm{A}^{*}$ algorithm is $112.62^{\circ}$, and the path length is $9.84 \mathrm{~m}$. According to the experimental results, compared with the primary $\mathrm{A}^{*}$ algorithm, the secondary $\mathrm{A}^{*}$ algorithm can effectively reduce the path length and unnecessary turning of the mobile robot during the travel. The path length is reduced by $2.21 \%$ and the turning angle is reduced by $37.43 \%$. The secondary $\mathrm{A}^{*}$ algorithm has effectively improved the turning angle of the mobile robot and has greatly optimized the travel path of the mobile robot. Conclusion: The improved $\mathrm{A}^{*}$ algorithm in this paper can effectively plan the travel path of the mobile robot, and ensure the efficiency and correctness of the planned path. The path planning and design error of the mobile robot in this paper is relatively low, and it can perform better path planning.
\end{abstract}

Keywords: Embedded Real-time System; Intelligent Robot; A* Algorithm; Robot Travel Path.

\section{Introduction}

Due to the development and progress of the times, people's demand for mobile robots has gradually increased, such as sweeping robots commonly found in households. It makes scholars' research on robots more in-depth. The travel path of mobile robots has always been one of the important researches carried out by scholars [1].

In recent years, due to the rapid development of mobile robots as well as their software and hardware technologies, there are more theoretical and practical researches on mobile robots. It makes the path planning of mobile robots and the tracking of their trajectories become the subject of scramble for researchers [2].

During the progress of the times, more and more mobile robots have been developed. There are various implementation methods for just the movement of the robot, such as mobile robots based on bionics similar to animals or humans, mobile robots that roll by wheels, and robots traveling by crawler drive. However, compared with other types of mobile robots, mobile robots that use wheels to move and change directions move faster and work more efficiently.
Therefore, scholars in robotics research have begun to focus on this type of mobile robot and carried out a detailed analysis and research. Since the research and design of ELMER robot by Professor W. Gray Walter of the University of Bristol in 1948, scholars and researchers' study on wheeled mobile robots has been carried out rapidly and achieved good results [3].

As far as current research is concerned, combining artificial intelligence and mobile robot research is an inevitable result of technological development. However, the research on the path problem of mobile robots alone and combined with artificial intelligence algorithms is relatively rare [4].

Some scholars have improved the ant colony algorithm and applied it to the path calculation and planning of mobile robots. Based on the strength of the pheromone, the probability of the heuristic factor, and the solution of the improved ant colony algorithm that excludes the construction of ants, proceed accordingly, the number of individuals in the entire algorithm process will gradually decrease. When the previous cycle reaches a preset standard or regulation, it will be forced to enter the next cycle. Therefore, this method may have many individuals who have not found a food source, and the accuracy 
of the pheromone update process is low, and the number of inaccurate updates is high [5].

In some studies, the ant colony algorithm and the chaos algorithm are combined. This combination of the two algorithms improves the defect of the low accuracy of the ant colony algorithm, and effectively expand the search range and search diversity. Compared with the ant colony algorithm, the final accuracy of the method has been greatly improved. While improving the algorithm, a new path planning method of the mobile robot is introduced to improve the efficiency of the algorithm and the accuracy of path planning [6].

To study the optimal travel path of the robot, this paper applies the embedded system to the path planning of the mobile robot, uses it as the processor of the mobile robot, and studies the path planning of the mobile robot and the algorithm to avoid obstacles. This paper proposes an improved $A^{*}$ algorithm. It will build a specific environment grid based on existing environmental materials. Using the improved $A^{*}$ algorithm, each node can be analyzed in detail. Then, the most suitable and fastest path is explored based on it.

\section{Methods}

\subsection{Embedded System}

Embedded system is a special-purpose computer system that is application-centric, computer technology-based. The software and hardware can be cut, and it is suitable for application systems.

The system has strict requirements on function, reliability, cost, volume, and power consumption [7].

Its main features are embedded and dedicated. Embedded devices are often used in "specific" situations and have the following characteristics compared to "generic" personal computers. Software and hardware can be cut, that is, when a certain function is not needed, the corresponding software and hardware can be removed.

The requirements for equipment functions, energy consumption, reliability, and cost are high. Similar to mobile phones, when the hardware configuration of the mobile phone is selected, the performance of the mobile phone processor is specified. Whether the operation of the mobile phone conforms to the behavioral logic of the human body, whether the menu settings of the mobile phone are efficient, and whether the functions of the mobile phone are complete and diverse are entirely dependent on the software. It requires the cooperation of drivers and applications to maximize the performance of the hardware [8].

The embedded Linux system is divided into user space and kernel space [9].

As shown in Figure 1, user space includes user applications and C libraries. Kernel space includes a system call interface, kernel, and architecturedependent kernel code.

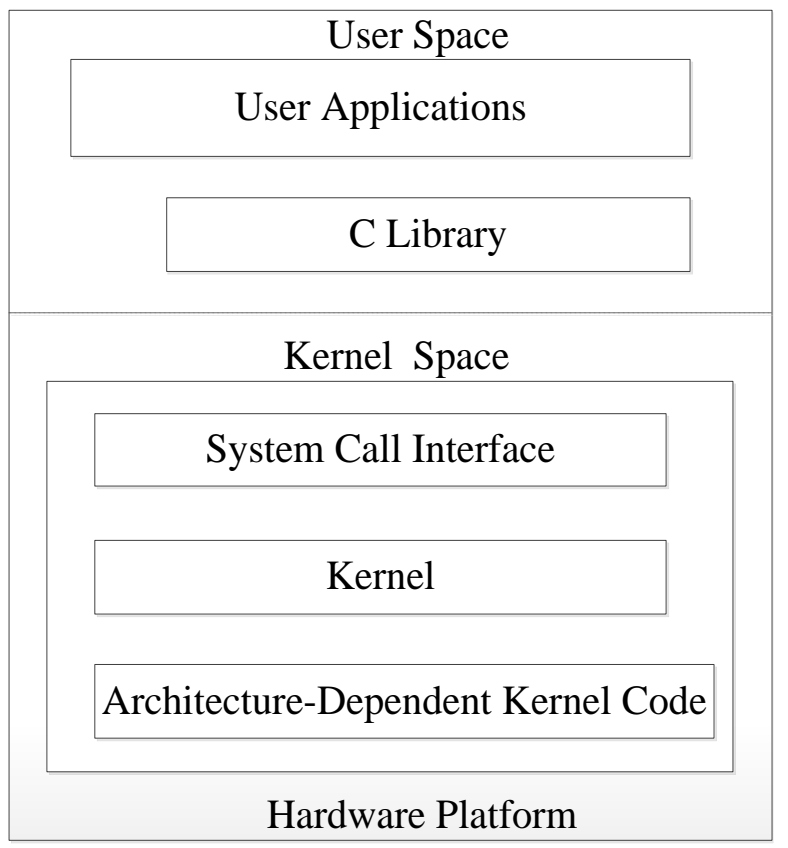

Figure 1: Linux architecture

\subsection{Establishment of the grid environment model}

In the establishment of the rasterization model of the environment, the grid size established is closely related to the accuracy of the travel path of the mobile robot. If the planned grid size is small, the resolution of the environment will be greatly improved. However, too small grid size will lead to too many grids, which makes the calculation of the model difficult, increases the processing load of the computer, and greatly lengthen the processing time of the computer. If the grid size is too large, the accuracy of the path planning will be reduced. Therefore, detailed calculations are required to obtain a suitable grid size.

The calculation of the grid size is shown in equations (1) and (2).

$$
\begin{aligned}
& l=\max \left(l_{s}, l_{\min }\right) \\
& l_{s}=\frac{S_{o b s}}{S} \times l_{\text {max }}
\end{aligned}
$$

Where: $l_{\min }=\min \left(l_{\text {obsmin }}, 2 R\right), l_{\text {obsmin }} \quad$ is the shortest side length of the obstacle. The distance between the two wheels of the mobile robot is $2 \mathrm{R}$. S is the area value of the environment under study. $\mathrm{S}_{\text {obs }}$ is the volume of the obstacle. $l_{\max }$ is the maximum value of the side length of the obstacle.

The mobile robot is likened to a movable point on a two-dimensional plane. In this way, the travel route of the mobile robot can be drawn as a flat graphic. The environment map consists of the grid $\operatorname{map}_{\mathrm{ij} .}$

$$
\text { map }=\left\{\operatorname{map}_{i j}, \operatorname{map}_{i j}=0,1,2,3\right\}
$$


Where: $\operatorname{map}_{i j}=0$ indicates that the unit grid is the starting position of the robot. $\operatorname{map}_{i j}=1$ indicates that the grid here is barrier-free. $\operatorname{map}_{i j}=2$ indicates the grid here is the range of the obstacle. $\operatorname{map}_{i j}=3$ indicates that the grid here is the range of the target.

After two-dimensional graphics transformation of the travel path of the mobile robot, the trajectory planning problem of the robot can be seen as the best route problem to avoid obstacles in a plane.

\section{$2.3 \mathrm{~A}^{*}$ algorithm}

The $A^{*}$ algorithm is a typical heuristic search algorithm of artificial intelligence. It can check all the nodes in the grid and use the evaluation function to find a more suitable travel path. In the process of the $\mathrm{A}^{*}$ algorithm, two sets of holding search information will be established, the OPEN table and the CLOSE table.

The OPEN table will store the nodes that have not yet been searched, and the CLOSE table will store the nodes that have been checked as well as the position and range information of the obstacles that have been checked. In the process of searching for the path node, the $A^{*}$ algorithm is developed from the eight directions of the selected node to obtain the child nodes [10].

The expansion order depends on the calculation of node generated values, which determines the search order of the nodes to be checked in the OPEN table, thereby finding the target node [11].

The expression of the valuation function is shown in equation (4).

$$
f(n)=g(n)+h(n)
$$

Where: $\mathrm{n}$ represents the node that is currently being checked. $\mathrm{f}(\mathrm{n})$ represents the evaluation function of node $n$. $g(n)$ represents the cost value from the first checked node to $n$. $h(n)$ is called the heuristic function, which represents the estimated cost value from the current node $\mathrm{n}$ to the target point. $\mathrm{h}(\mathrm{n})$ uses Euclidean distance, which has the following definition.

$$
h(n)=\sqrt{\left(x_{n}-x_{\text {goal }}\right)^{2}+\left(y_{n}-y_{\text {goal }}\right)^{2}}
$$

Where: $\left(x_{n}, y_{n}\right)$ represents the center coordinates of the grid where the current node is located. $\left(x_{\text {goal }}, y_{\text {goal }}\right)$ represents the center coordinates of the grid where the target point is located.

The initial point of the robot is defined as the node $\mathrm{S}$, and the minimum value obtained by the total cost $f(n)$ corresponds to the node $N$. The child node extended by the node $\mathrm{N}$ is called $\mathrm{M}$, and the target node is 0 . The overall planning flowchart of $A^{*}$ algorithm is shown in Figure 2.

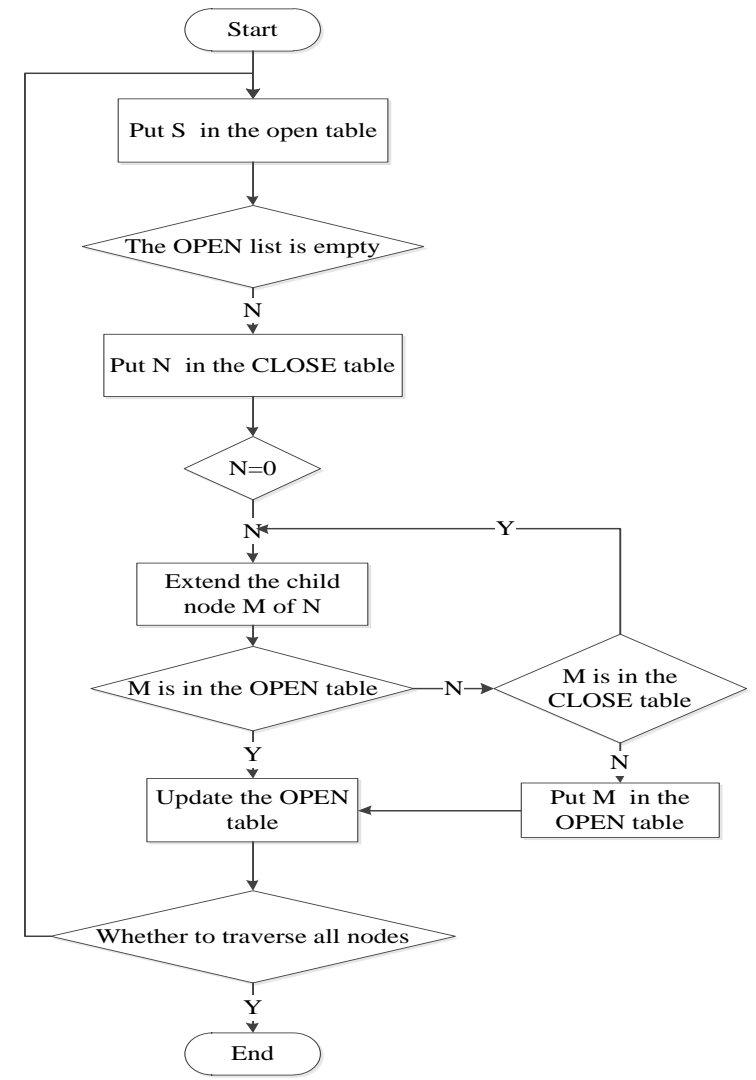

Figure 2: A* algorithm flowchart

\subsection{Path planning based on $A^{*}$ algorithm}

When the traditional $\mathrm{A}^{*}$ algorithm is used to plan the travel route of a mobile robot, there will be too many redundant points in the travel route data obtained. And the planned route data has many unnecessary turning times and excessive turning angles. Therefore, to solve the problems existing in the traditional algorithm, this paper has made necessary improvements to the traditional $A^{*}$ algorithm. Based on the original $A^{*}$ algorithm, a secondary $A^{*}$ search algorithm is performed. The turning points planned in the previous $A^{*}$ algorithm and the originally set starting and ending points are retained. This method can effectively reduce or even remove redundant points in robot path planning. It can shorten the length of the route that the robot needs to travel from the start point to the end point without causing the robot to hit an obstacle, decrease the turning times, and reduce the angle of adjustment required for turning.

The working principle of the improved $A^{*}$ algorithm is as follows.

First, the primary $A^{*}$ algorithm is performed and the first travel path planning of the mobile robot is performed. Also, the planned data is saved. In the path planning process, each path node that is planned and the nodes that are not adjacent to it are used as extension points. Then, whether to re-form a new path is determined based on the magnitude of the evaluation function and whether the link 
between the two path nodes passes through the obstacle. If the evaluation function is less than the initial value and the link between the two path nodes does not pass through the obstacle, the two path nodes are connected, and the intermediate path nodes are deleted to form a new path. Conversely, no changes are made to the path.

Finally, the shortest path is generated that contains only the starting position, the turning point position, and the target position.

\section{Results and Discussion 3.1 Acquisition of the initial path}

The simulation comparison experiments are performed on the path data obtained by the original $\mathrm{A}^{*}$ algorithm and the improved $\mathrm{A}^{*}$ algorithm. The simulation comparison is performed on a grid map of $10 \mathrm{~m} * 10 \mathrm{~m}$. The rectangle in the figure indicates the static obstacle, and the circle indicates the starting position of the robot. The square indicates the target position, and the small circle indicates the selected path point on the path.

The simulation results are shown in Figure 3 and Figure 4.

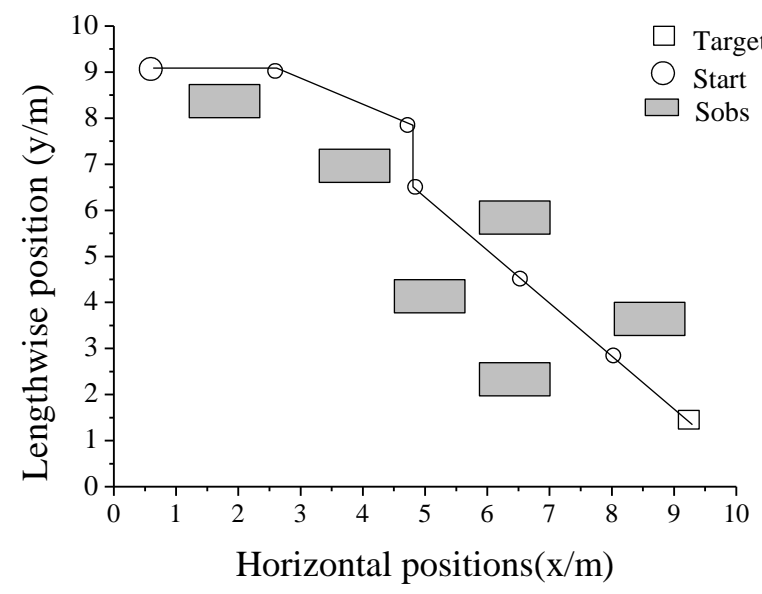

Figure 3: Primary $A^{*}$ algorithm optimization

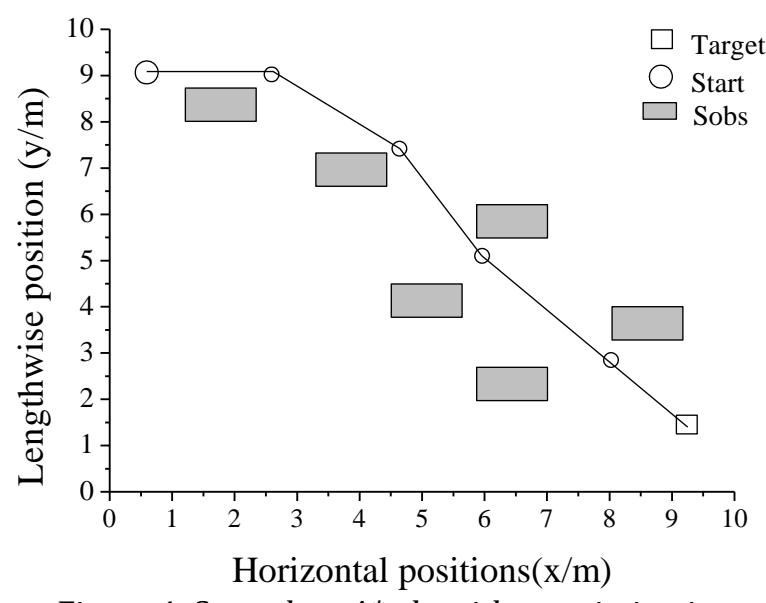

Figure 4: Secondary $A^{*}$ algorithm optimization
From Figure 3 and Figure 4, the total turning angle of the mobile robot using the primary $A^{*}$ algorithm is $180^{\circ}$ and the path length is $10.07 \mathrm{~m}$.

The total turning angle of the mobile robot using the secondary $\mathrm{A}^{*}$ algorithm is $112.62^{\circ}$, and the path length is $9.84 \mathrm{~m}$. According to the experimental results, compared with the primary $A^{*}$ algorithm, the secondary $A^{*}$ algorithm can effectively reduce the path length and unnecessary turning of the mobile robot during the travel. The path length is reduced by $2.21 \%$ and the turning angle is reduced by $37.43 \%$. The secondary $A^{*}$ algorithm has effectively improved the turning angle of the mobile robot, and has greatly optimized the travel path of the mobile robot.

The grid method is used to divide the environment that the mobile robot needs to simulate into a grid of $250 \mathrm{~mm} \times 250 \mathrm{~mm}$ size.

In the grid environment map constructed in this paper, two different sets of starting points and target points are selected, and the proposed improved $A^{*}$ algorithm is used to carry out path planning experiments for mobile robots. The moving cost is stored in the obstacle grid as 300 , and the moving cost is stored in the blank free grid as 10 .

The size of the mobile robot is $500 \mathrm{~mm} \times 500 \mathrm{~mm}$, and the grid size is $250 \mathrm{~mm} \times 250 \mathrm{~mm}$. Therefore, to prevent the planned path from approaching the obstacle, the moving cost is stored in the two rows of grids next to the obstacle as 200. The heuristic function is the improved $\mathrm{h}(\mathrm{n})=4^{*}$ abs (goalpoxnewrow)+3*abs(goalposy-newcol). Where newrow is the abscissa of the current node and newcol is the ordinate of the current node. goalpox is the abscissa of the target node, and goalposy is the ordinate of the target node. The results of the path planning experiment are shown in Figure 5.

The circle indicates the starting point and the asterisk indicates the ending point.

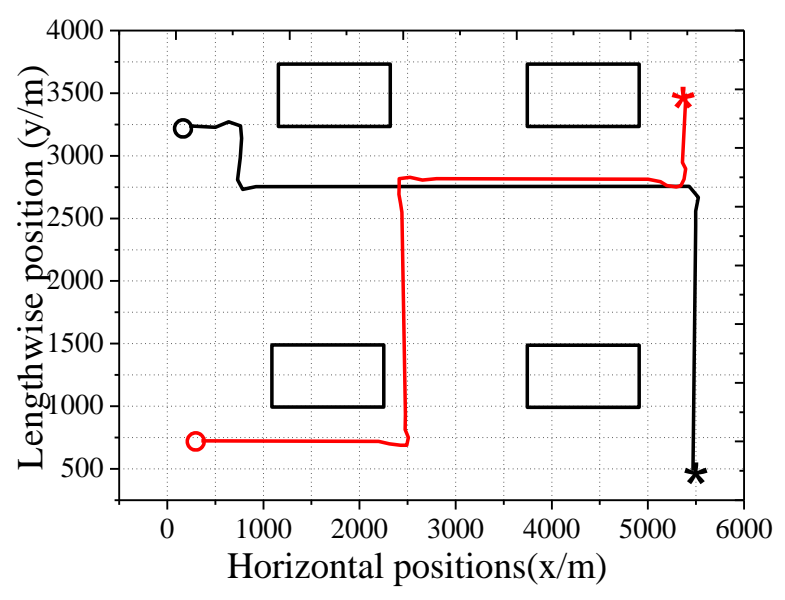

Figure 5: Experimental results of mobile robot path planning 
As can be seen from the above figure, the method in this paper is effective in the planning phase of the path.

It can plan a short path without obstacles for the mobile robot.

\subsection{Results of mobile robot tracking planning path}

Based on the experimental results of mobile robot path planning, the designed control algorithm is used to make the mobile robot track the planned trajectory.

The direction angle of the planned expectation path to the right is set to 0rad, and the direction angle of the planned expectation path to the left is set to $-\pi$. The direction angle of the planned expectation path to the upward is set to $\pi / 2$ and the direction angle of the planned expectation path to the downward is set to $-\pi / 2$.

In the planned path tracking experiment, the error between the initial position and the actual initial position of the mobile robot's expected trajectory is 0 .

Taking the parameters $\mathrm{k} 1=0.2, \mathrm{k} 2=2, \mathrm{k} 3=45, \mathrm{k} 4=5$ in the parameter control algorithm, the experimental results of the mobile robot tracking the planned path are shown in Figure 6.

The black and red are the expected paths of the mobile robot.

The blue and green are the actual paths of the mobile robot.

The circle indicates the starting position, and the asterisk indicates the end position.

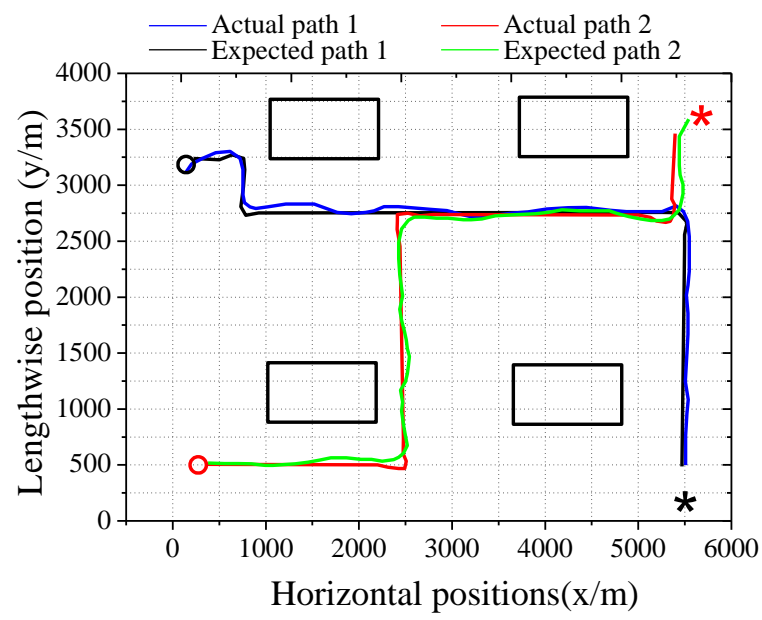

Figure 6: The actual path of the mobile robot planning path at different starting points and target points

In two different path experiments, the traveling error of the mobile robot in various directions and the tracking error of the target point are shown in Figure 7.

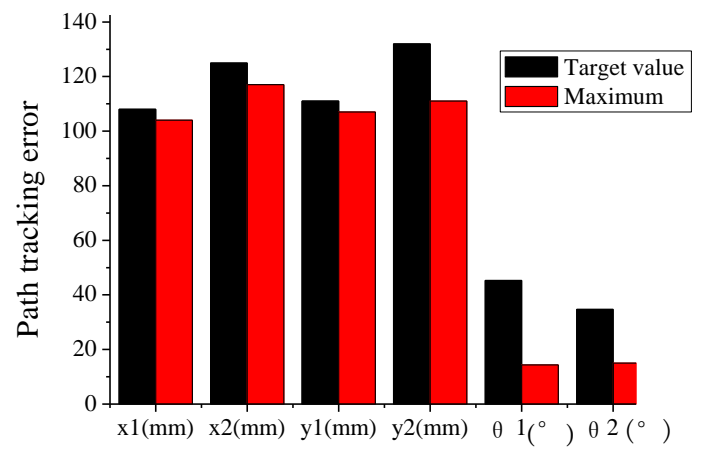

Figure 7: Mobile robot path tracking error

From the path tracking error of the mobile robot shown in the figure, it can be seen that after increasing the expected path of the mobile robot, the mobile robot can perform good path tracking.

However, the traveling error of the mobile robot increases, and the error value at the starting point is the largest. It is due to the accumulation of errors caused by the increase of the path.

\subsection{Tracking control results of the path around the map}

To check whether the travel of the mobile robot meets the requirements under different path conditions, a longer path is designed for research.

The designed starting position is $(250,500)$ and the target position is $(1250,500)$. In the experiment, the error between the expected initial position and the actual initial position of the mobile robot is 0 .

The mobile robot path tracking results are shown in Figure 8. The black trajectory is the expected path planned by the mobile robot, and the red trajectory is the actual moving path of the mobile robot.

The circle is used to indicate the starting position of the mobile robot and the asterisk is used to indicate the end position of the mobile robot.

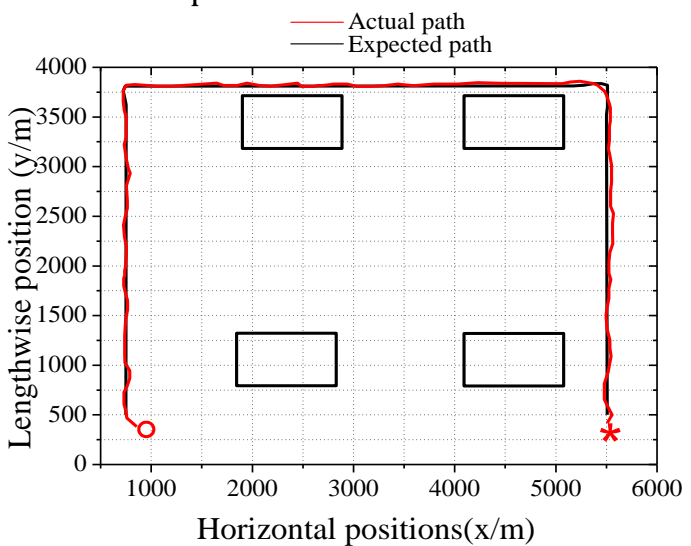

Figure 8: Experimental results for the mobile robot path tracking of the expected path around the map

After conducting the same experiment 6 times to determine the tracking effect of the mobile robot on the path, it is found that the mobile robot can track the expected path in the paper relatively well. 
In the expected path around the whole field, the path tracking error of the mobile robot is shown in Figure 9.

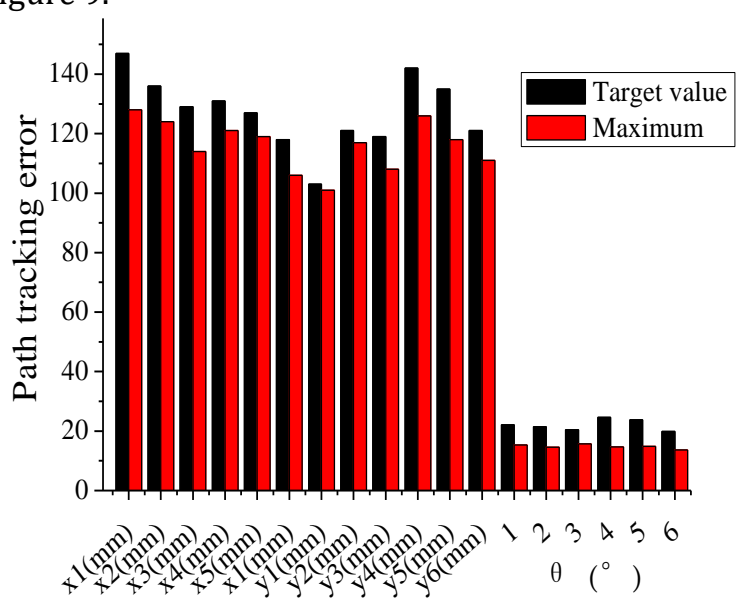

Figure 9: Mobile robot path tracking error

From the path tracking error of the mobile robot shown in the figure, it can be seen that after increasing the expected path of the mobile robot, the mobile robot can perform good path tracking. However, when the expected path is increased, the travel error of the mobile robot also increases. It is due to the accumulation of errors caused by the increase of the path. From the experimental results of the mobile robot tracking different paths, it can be known that the mobile robot path tracking algorithm designed in this paper has a good effect and the potential for further development.

\section{Discussion}

Due to the rapid development of mobile robots as well as their software and hardware technologies, there is more research on the theory and practice of mobile robots, which makes the path planning of mobile robots and the tracking of their trajectories become the subject of scramble for researchers [12]. Therefore, this paper has carried out a series of researches on the path planning of mobile robots.

The paper first introduces the required technology and research methods. Then, it applies embedded technology to the mobile robot path planning. Based on the original $A^{*}$ algorithm, the secondary $A^{*}$ search algorithm is performed. The turning points planned in the previous $A^{*}$ algorithm and the originally set starting and ending points are retained. This method can effectively reduce or even remove redundant points in robot path planning. It can shorten the length of the route that the robot needs to travel from the start point to the end point without causing the robot to hit an obstacle, decrease the turning times, and reduce the angle of adjustment required for turning. After simulation experiments, the final result is obtained: the improved $\mathrm{A}^{*}$ algorithm has a good effect on the path planning problem of mobile robots, and it can plan the most suitable and efficient path for mobile robots.

\section{Conclusions}

The path problem of the mobile robot is an essential and important step in the research of mobile robot. Therefore, this paper studies the travel path planning of mobile robots.

After the improvement of the research algorithm in this paper, an effective method of the path planning of the mobile robot is obtained, which can move along the path planned in this paper (the error is within the acceptable range). After simulation research, the trajectory suitable for the mobile robot is obtained. Thus, this research has certain practicability.

However, the simulation research conducted in this paper is only in the theoretical stage and the research scenario is relatively simple. Therefore, there is still a phenomenon that cannot be in line with the actual situation.

The scenarios studied in this paper are far less complicated than the scenarios that mobile robots need to work on, such as sweeping robots that need to work in complex home environments.

For mobile robots, obstacles are complex and vary in size. Thus, the future research will be based on this research, to simulate the actual scene, and discuss whether the improved algorithm in this paper has the capability of practical application.

\section{References}

[1] T. H.Wu, A. Y. Wang, N. Oros et al., "Adaptive Robot Path Planning Using a Spiking Neuron Algorithm With Axonal Delays," IEEE Transactions on Cognitive and Developmental Systems, vol. 10, no. 2, pp. 126-137, 2018.

[2] H. Qu, L. W. Huang, and X. Ke, "Research of improved ant colony based robot path planning under dynamic environment, "Dianzi Keji Daxue Xuebao/Journal of the University of Electronic Science and Technology of China, vol. 44, no. 2, pp.260-265, 2015.

[3] X. F. Wan, W. Hu, B. J. Zheng et al., "Robot path planning method based on improved ant colony algorithm and Morphin algorithm, "Science \& Technology Review, vol. 33, no. 3, pp.84-89, 2015.

[4] P. K. Das, H. S. Behera, P. K. Jena et al., "MultiRobot Path Planning in a dynamic environment using Improved Gravitational Search Algorithm" Journal of Electrical Systems \& Information Technology, vol. 3, no. 2, pp. 295$313,2016$.

[5] T. Kurosaka, and M. Kaneko, "Autonomous Mobile Robot Selecting Optimum Path with Safe Speed Control in Consideration of Blind Area of 
Vision Sensors, "IEEJ Transactions on Electronics Information and Systems, vol. 135, no. 4, pp. 356-364, 2015.

[6] X. Xu, Y. Guan, B. Hong et al., "A humanoid robot path planning method based on virtual forcedirected particle swarm optimization, "International Journal of Wireless and Mobile Computing, vol. 9, no. 4, pp. 325, 2015.

[7] K. Charalampous, A. Amanatiadis, and A. Gasteratos, "Efficient Robot Path Planning in the Presence of Dynamically Expanding Obstacles, "Lecture Notes in Computer Science, vol. 7495, no. 1,pp. 330-339, 2012.

[8] X. Q. Xu, and Q. B. Zhu, "Multi-artificial fishswarm algorithm and a rule library based dynamic collision avoidance algorithm for robot path planning in a dynamic environment, "Tien Tzu Hsueh Pao/Acta Electronica Sinica, vol. 40, no. 8, pp. 1694-1700, 2012.
[9] D. W. Gong, N. Geng, and Y. Zhang, "Robot path planning in environment of many terrains, "Kongzhi yu Juece/Control and Decision, vol. 27, no. 5, pp. 708-712, 2012.

[10] K. Charalampous, A. Amanatiadis, and A. Gasteratos, "Efficient Robot Path Planning in the Presence of Dynamically Expanding Obstacles, "Lecture Notes in Computer Science, vol. 7495, no. 1, pp. 330-339, 2012.

[11] R. Kala, A. Shukla, and R. Tiwari, "Dynamic Environment Robot Path Planning Using Hierarchical Evolutionary Algorithms, "Cybernetics and Systems, vol. 41, no. 6, pp. 435-454, 2010.

[12] D. L. Luo, and S. X. Wu, "Ant colony optimization with potential field heuristic for robot path planning, "Xi Tong Gong Cheng Yu Dian Zi Ji Shu/Systems Engineering and Electronics, vol. 32, no. 6, pp. 1277-1280, 2010. 